\title{
Baryon Acoustic Oscillation Intensity Mapping as a Test of Dark Energy
}

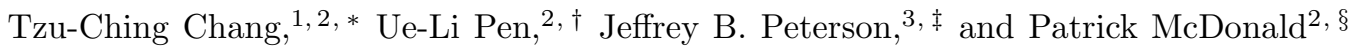 \\ ${ }^{1}$ Inst. for Astronomy and Astrophysics, Academia Sinica, PO Box 23-141, Taipei 10617, Taiwan \\ ${ }^{2}$ CITA, University of Toronto, 60 St.George St., Toronto, ON, M5S 3H8, Canada \\ ${ }^{3}$ Department of Physics, Carnegie Mellon University, 500 Forbes Ave, Pittsburgh, PA 15213, USA
}

\begin{abstract}
The expansion of the universe appears to be accelerating, and the mysterious anti-gravity agent of this acceleration has been called "dark energy". To measure the dynamics of dark energy, Baryon Acoustic Oscillations (BAO) can be used. Previous discussions of the BAO dark energy test have focused on direct measurements of redshifts of as many as $10^{9}$ individual galaxies, by observing the $21 \mathrm{~cm}$ line or by detecting optical emission. Here we show how the study of acoustic oscillation in the $21 \mathrm{~cm}$ brightness can be accomplished by economical three dimensional intensity mapping. If our estimates gain acceptance they may be the starting point for a new class of dark energy experiments dedicated to large angular scale mapping of the radio sky, shedding light on dark energy.
\end{abstract}

Introduction.- To understand dark energy and sharply test theories of its character, it is necessary to precisely measure the last half of the expansion history. This period corresponds to the redshift range $0<z \lesssim 2$. Many techniques have been proposed to study the latestage expansion history, and some of the most promising make use of Baryon Acoustic Oscillations (BAO) 1]. In addition to producing CMB structure, the acoustic oscillations also produced density structure in the atomic gas and dark matter, which is still detectable today. Many groups have reported 2 to $3 \sigma$ detections, in the low redshift universe, of periodic structures in the density of galaxies at the predicted wavelengths across the sky[2]. Because the acoustic waves are frozen in after recombination, the BAO peak wavelengths can be used as a cosmological standard ruler: observation of the angular size of the peak wavelengths across a range of redshifts allows accurate measurement of the expansion history.

In this letter we present calculations of structure in the three dimensional brightness due to the hyperfine transition of neutral hydrogen (HI) at $21 \mathrm{~cm}$ wavelength. We show that, via $21 \mathrm{~cm}$ emission, baryon oscillations could be precisely measured, using a telescope just 200 wavelengths in diameter, since each cosmic cell of the appropriate scale contains more than $10^{12} M_{\odot}$ of emitting neutral hydrogen gas. We present forecasts on the dark energy constraints from this new type of observation, which are competitive with the best proposed dark energy experiments 3]. Where needed in the analysis we adopt $W M A P 3$ values for the cosmological parameters 4 , $\Omega_{m}=0.24, \Omega_{b}=0.04, \Omega_{\Lambda}=0.76$, and $h=0.73$, where $\Omega_{m}, \Omega_{b}$, and $\Omega_{\Lambda}$ are the matter, baryon, and dark energy fractional density, respectively, and $h$ is the dimensionless Hubble parameter. We also use the WMAP3 error limits for these values.

A recent paper proposed a test of dark energy models by measurement of baryon oscillations in the $21 \mathrm{~cm}$ brightness field at redshifts $z>3[5]$. Using only such high redshift data two cosmological parametersdepartures of spatial flatness $\Omega-1$ and a slow change of dark energy equation of state - are nearly indistinguish- able. It is only when precise flatness $\Omega=1$ is assumed that such high redshift data can be used to constrain dark energy models. In this letter we use the more standard approach and assume that $\Omega$ is not perfectly measured. Then data at lower redshift become essential if dark energy models are to be tightly constrained.

Detectability. - To measure the oscillations it is not necessary to detect individual galaxies, but only to measure the variation in HI mass on large scales. A familiar analogy is the study of galaxies via images of their surface brightness. Except for the nearest of galaxies, we do not detect the individual stars that produce the optical emission, indeed the pixels of most galaxy images represent the emission of millions of stars. Never-the-less such images allow study of galactic structure.

Above the third BAO peak nonlinear evolution attenuates BAO structure, so the third peak is at the smallest spatial scale we need consider. This peak has a wavelength $35 h^{-1}$ Mpc. A Nyquist sampled map therefore needs pixels of size $18 h^{-1} \mathrm{Mpc}$. At $z=1.5$ the corresponding angular wavelength is 20 arc minutes, which requires a telescope of approximately 200 wavelength, or 100 meters, to resolve.

We base our estimate of neutral hydrogen mass contained in a $18 h^{-1} \mathrm{Mpc}$ volume $\left(M_{\mathrm{HI}} \sim 2 \times 10^{12} M_{\odot}\right)$ on Ly $\alpha$ absorption studies of the cosmic density of neutral gas [6], which find $\Omega_{\mathrm{HI}} \sim 1 \times 10^{-3}$ at $z \sim 1$, with a weak dependence on redshift.

The average sky brightness temperature due to the $21 \mathrm{~cm}$ line is about $300 \mu \mathrm{K}$. The mean brightness temperature $T_{b}$ of the $21 \mathrm{~cm}$ line can be estimated using [7]

$$
T_{b}=0.3\left(\frac{\Omega_{\mathrm{HI}}}{10^{-3}}\right)\left(\frac{\Omega_{m}+a^{3} \Omega_{\Lambda}}{0.29}\right)^{-1 / 2}\left(\frac{1+z}{2.5}\right)^{1 / 2} \mathrm{mK} .
$$

Most observation of the sky are measurements of variations in temperature $\Delta T_{b} \sim T_{b} \delta$, where $1+\delta=\rho_{g} / \bar{\rho}_{g}$ is the normalized neutral gas density, and $a=(1+z)^{-1}$ is the scale factor.

On $18 h^{-1}$ Mpc scales spatial variations of the sky brightness due to $21 \mathrm{~cm}$ emission in the cosmic web 


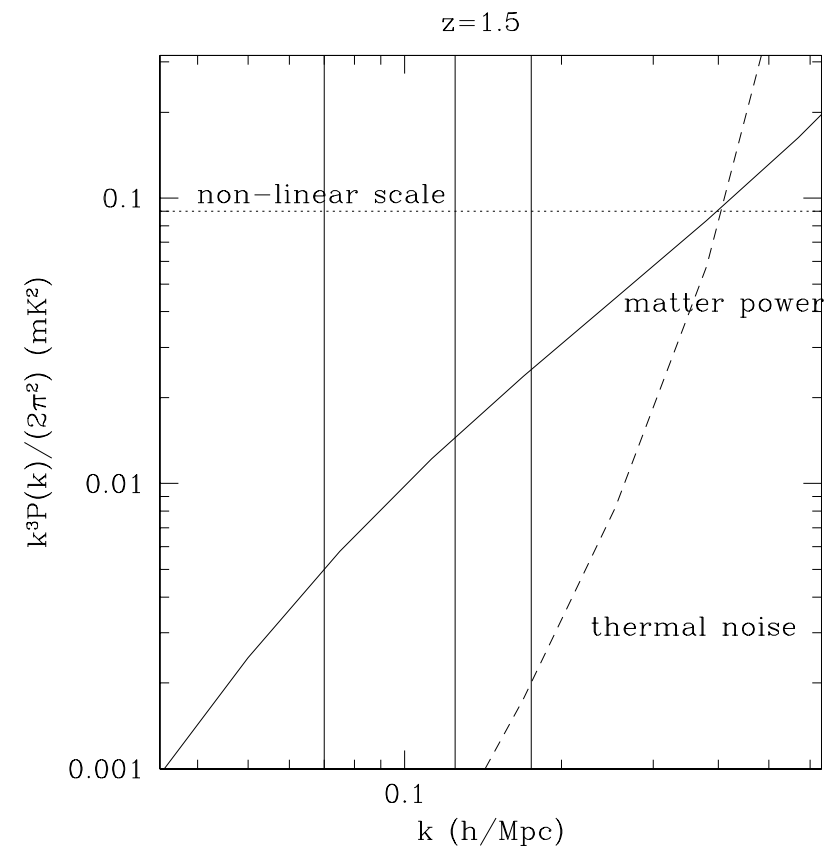

FIG. 1: The power spectrum of neutral hydrogen hyperfine emission at $z=1.5$ is shown by the solid line. The dashed line indicates the map noise from a 200-by-200-meter radio cylinder telescope operated in transit mode for 100 days, assuming a telescope system temperature of $50 \mathrm{~K}$. The horizontal dotted line indicates the non-linear scale at $z=1.5$. The vertical lines indicate the location of peaks in Figure 2

structure presents a sky noise at the $150 \mu \mathrm{K}$ level. Figure 1 shows the amplitude of this cosmological large scale structure, while Figure 2 plots the small oscillations imprinted by BAO. The variation of gas density due to baryon oscillations is small compared to the fluctuation of the large-scale structure. To detect the BAO signals beneath this noise Fourier analysis of a large regions of sky is required. The observations should cover as much sky as possible.

For a filled aperture telescope the RMS variation in measured sky temperature $\Delta T=T_{\text {sys }} / \sqrt{\Delta f t_{\text {int }}}$, where $\Delta T$ is the temperature in Kelvin, $T_{\text {sys }}$ is the system temperature of the radio telescope in Kelvin, $\Delta f$ is the observing bandwidth in Hertz and $t_{\text {int }}$ the integration time in seconds. Assuming current technology, which allows $T_{\text {sys }}=50 \mathrm{~K}$, one needs an observing bandwidthtime product of $2 \times 10^{11}$ to bring the thermal map noise down to $\sim 100 \mu \mathrm{K}$, below the cosmic structure noise. In frequency space $18 h^{-1} \mathrm{Mpc}$ spans roughly $3 \mathrm{MHz}$ at $z=1.5$, so the required observation time is $\sim 6 \times 10^{4}$ seconds, about 18 hours per pixel. Small regions of sky might be mapped at this noise level using existing telescopes such as the Green Bank Telescope (GBT), the Effelsberg 100-meter Radio Telescope or the Parkes Telescope.

Brightness sensitivity is not improved by increase of

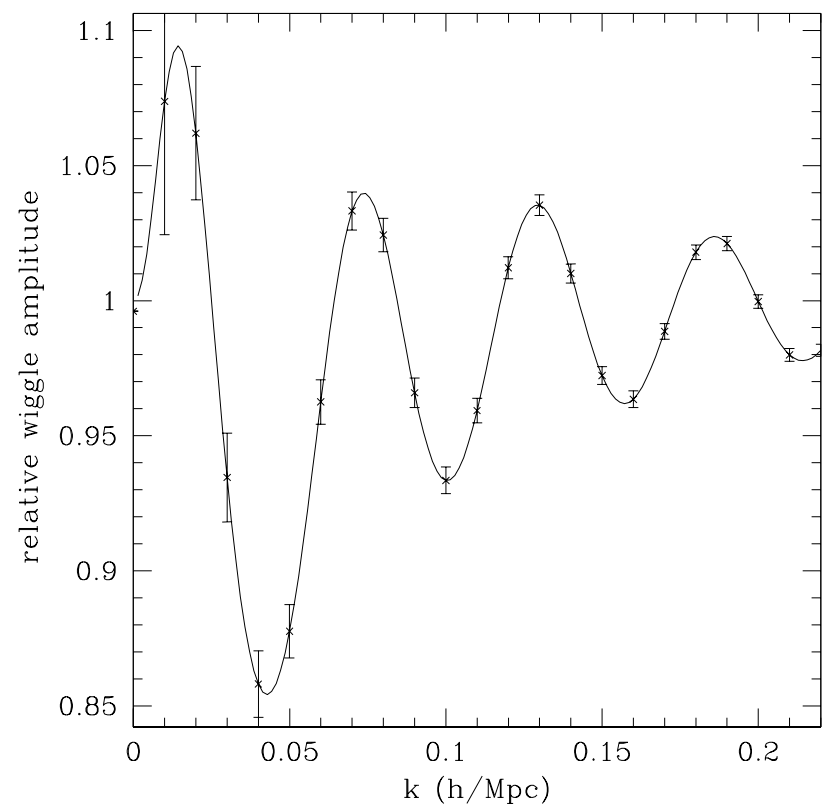

FIG. 2: Baryon Acoustic Oscillations. To show BAO we plot the ratio of matter power spectrum that contains contributions from baryons, calculated using a fitting formula [10], over a power spectrum with no baryons. To single out the oscillation, we further divide the ratio by a smooth fitting curve to the overall spectrum. The error bars represent projections of the sensitivity possible with a 200-by-200-meter cylinder telescope.

telescope aperture, instead multiple receivers, either on a single reflector or on individual reflectors, can be used to increase the accumulated integration time.

For sensitivity estimates, we consider an intensity mapping telescope, dubbed IM, covering a square aperture of size $200 \mathrm{~m} \times 200 \mathrm{~m}$. We subdivide this area into 16 cylindrical sections, each $12.5 \mathrm{~m}$ wide and $200 \mathrm{~m}$ long, analogous to the Hubble Sphere Hydrogen Survey (HSHS) 11]. The cylinders are aligned North-South allowing the telescope to instantaneously sample almost the entire meridian. This geometry allows all-sky coverage each 24 hour period in a telescope with no moving parts. The estimated sensitivity of the survey is shown in Figure 1.

Foregrounds. - The desired BAO signal is smaller than other sources of sky brightness structure. The largest interference is due to synchrotron emission from the Galaxy. The spatial structure of this emission exceeds the BAO signal by $10^{3}$. Similar emission by extragalactic radio sources distributed across the sky will contribute sky brightness fluctuations on smaller scales. However all these foregrounds are spectrally smooth, with brightness temperature $T \propto f^{\alpha}$, so one expects to be able to subtract them spectrally. The image from each $3 \mathrm{MHz}$ slice of the frequency spectrum contains a unique BAO pattern, largely uncorrelated with the neighboring slices. 
In contrast, the foregrounds and backgrounds are highly correlated. By subtracting the average of both neighboring slices the BAO signal can be retained while the other emission is removed. Of course this subtractions will be imperfect, because of imperfections in the telescope and spatial variations in the astrophysical source spectrum. A similar challenge is faced by the higherredshift $21 \mathrm{~cm}$ experiments probing the Cosmic Reionization era and many foreground subtraction methods have been proposed (12] and references therein).

The spectral index $\alpha$ for Galactic emission varies across the sky. The frequency-double-difference technique described above suppresses the Galactic sky brightness but leaves a residual of order $\Delta T=\Delta \alpha(\Delta f / f)^{2} T$ to first power in $\Delta \alpha$, where $\Delta \alpha$ is the uncertainty in spectral index and $\Delta f$ the frequency difference. Using the WMAP3 measurement we estimate

$$
\Delta T \sim 1.3 \Delta \alpha\left(\frac{l}{200}\right)^{-0.5}\left(\frac{1+z}{2.5}\right)^{3.2}\left(\frac{\Delta f}{f}\right)^{2} \mathrm{~K},
$$

where $l$ is the spherical harmonic number. The normalization factor of 1.3 Kelvin temperature fluctuation is derived by extrapolating from the WMAP3 measurement of Galactic foreground variation at $20 \mathrm{GHz}$ on $3.7^{\circ}$ degree scale, along with the spectral index of synchrotron emission at mid-Galactic latitude [13]. Adopting $\Delta \alpha=1$, a conservative upper limit, we estimate a residual Galactic interference signal which is plotted in Figure 3 ,

Figure 3 shows various potential limitations to BAO measurements using $21 \mathrm{~cm}$ sky brightness mapping. The top exclusion comes from the non-linear scale, which erases the baryon wiggles. The left exclusion comes from the finite volume, which sets a lower bound on the lowest wavenumber that fits within a given volume. The lower right is a rough estimate of the impact of foregrounds using the double frequency difference described above. The shaded region reflects the region where the estimated residual foreground from Eqn. (2) exceeds the expected signal in Eqn. (11).

Parameter Estimation. - To estimate the sensitivity of this technique for measurement of dark energy parameters, we follow the procedure in Seo and Eisenstein (2007) [14] and carry out the standard figure-of-merit calculation used in the DETF report [3]. Our calculations include the effects of redshift space distortion and a simplified model of non-linearity using a smoothing window. This type of analysis, the conversion from survey data to cosmological parameters, is an area of active research, and several effects such as mode-mode coupling are still being investigated. Our use of shells of $21 \mathrm{~cm}$ surface brightness introduces two new types of unknowns into the analysis: the HI density, and the bias of $21 \mathrm{~cm}$ sources. To accommodate these uncertainties we make one sensitivity estimate using conservative values for the unknown quantities (IM) as our baseline model, and one with a

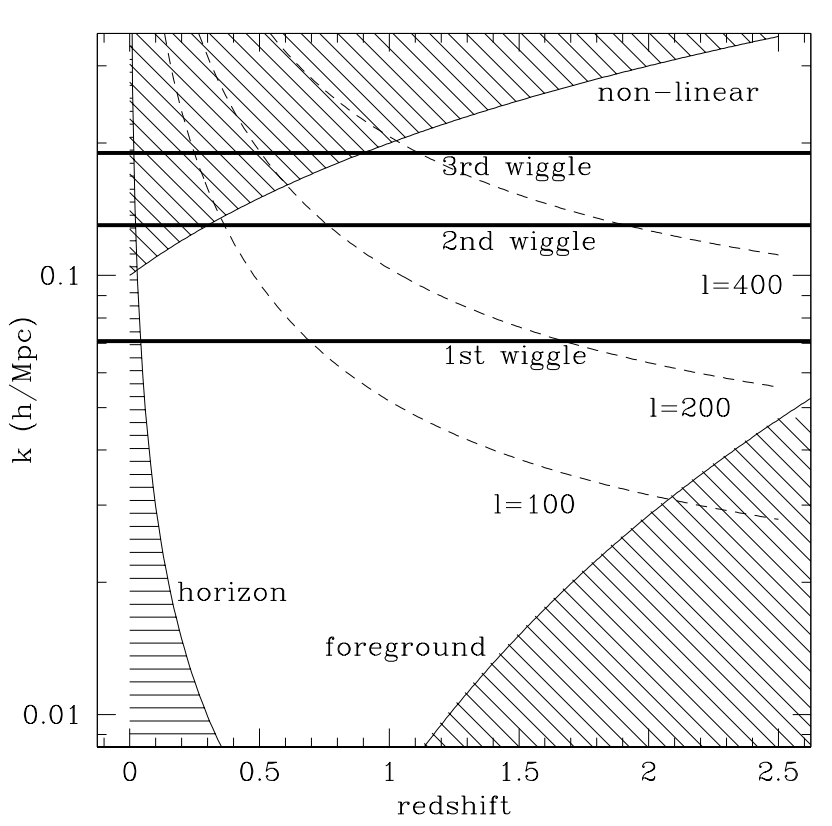

FIG. 3: The observable parameter space in redshift and in scale $(\mathrm{k})$ for BAO. The shaded regions are observationally inaccessible (see text). The horizontal lines indicate the scale of the first three BAO wiggles, and the dashed lines show contours of constant spherical harmonic order $\ell$.

worst case assumption that each of these effects works to increase the error (IMW). The IM case estimate assumes that $\Omega_{\mathrm{HI}}$ remains at the present day value of 0.0005 , independent of redshift, and we adopt a bias $b=1$. The shot noise from present day HI galaxies [15] is negligible. Motivated by the reconstruction of BAO degradation due to non-linearity in [16] the Seo and Eisenstein damping scale is shortened by a factor of two. For the worst case (IMW) estimate we let $\Omega_{\mathrm{HI}}$ to be 0.0004 . We adopt a bias $b=0.8$, which is the current estimate of the low $z$ value. This increases the noise, and seems securely to be a worst case because galaxies are unlikely to be both antibiased and rare. We assume that $21 \mathrm{~cm}$ sources are rare and massive at high redshift, and we use the Lyman-Break Galaxy luminosity function (with a density of $0.004 \mathrm{~h}^{3} \mathrm{Mpc}^{-3}$ ) for the shot noise calculation. The Seo and Eisenstein damping scale is assumed.

The time variable dark energy density is parametrized as $\rho_{D E} \propto(1+z)^{3(1+w)}$; an ordinary cosmological constant corresponds to $w=-1$, and the principal goal of dark energy experiments is to measure deviations of $w$ from -1 . We first determine the Fisher matrix for the tangential and radial distances errors. From these, we compute the errors on dark energy parameters. The resulting projected errors to the equation of state are shown in Figure 4 Table \ quantifies the errors, which can be compared to other dark energy surveys.

The mapping of cosmological large scale structure by 


\begin{tabular}{lcccccccccccccc}
\hline \hline & $\Omega_{m} h^{2}$ & \multirow{2}{*}{$\Omega_{b} h^{2}$} & $\Omega_{\mathrm{DE}}$ & $a_{p}$ & $w_{p}$ & $w^{\prime}$ & $\Omega_{k}$ & $\log _{10}(A)$ & $n_{s}$ & $h$ & $\Omega_{m}$ & FoM \\
reference value & 0.128 & 0.0223 & 0.760 & & -1.00 & 0.00 & 0.00 & -8.64 & 0.950 & 0.730 & 0.240 & \\
P+IM & 0.0012 & 0.00017 & 0.0058 & 0.65 & 0.015 & 0.16 & 0.00072 & 0.0077 & 0.0062 & 0.0075 & 0.0054 & 421.9 \\
P+IMW & 0.0012 & 0.00017 & 0.010 & 0.63 & 0.023 & 0.29 & 0.00084 & 0.0077 & 0.0062 & 0.015 & 0.010 & 152.3 \\
P+II & 0.0012 & 0.00017 & 0.012 & 0.79 & 0.036 & 0.52 & 0.0031 & 0.0076 & 0.0061 & 0.019 & 0.012 & 53.39 \\
P+II+III & 0.0011 & 0.00015 & 0.0045 & 0.76 & 0.020 & 0.18 & 0.0021 & 0.0063 & 0.0046 & 0.0070 & 0.0043 & 279.5 \\
P+II+III+IV & 0.00091 & 0.00013 & 0.0028 & 0.75 & 0.011 & 0.090 & 0.0013 & 0.0050 & 0.0028 & 0.0045 & 0.0026 & 975.2 \\
P+II+III+IV+IM & 0.00068 & 0.00013 & 0.0025 & 0.69 & 0.0082 & 0.068 & 0.00047 & 0.0049 & 0.0028 & 0.0032 & 0.0023 & 1793. \\
P+II+III+IV+IMW & 0.00071 & 0.00013 & 0.0027 & 0.71 & 0.0095 & 0.073 & 0.00063 & 0.0050 & 0.0028 & 0.0036 & 0.0025 & 1440. \\
\hline \hline
\end{tabular}

TABLE I: Fiducial cosmological parameters and error bars for combining the Planck mission with the fiducial BAO intensity mapping experiment such as IM $(\mathrm{P}+\mathrm{IM})$, with the Dark Energy Task Force[3] (DETF) Stage II projects (P+II), plus the Stage III projects $(\mathrm{P}+\mathrm{II}+\mathrm{III})$, plus the Stage IV projects $(\mathrm{P}+\mathrm{II}+\mathrm{III}+\mathrm{IV})$, and all above combined $(\mathrm{P}+\mathrm{II}+\mathrm{III}+\mathrm{IV}+\mathrm{IM})$. Here IM is the conservative best guess for intensity mapping experiment parameters, while IMW is the worse case scenario (see text). DETF Stage II is well-defined which includes weak lensing (WL), cluster evolution (CL), and supernova (SN) experiments; for Stage III we use the optimistic photometric versions of WL, CL, and SN; for Stage IV we use the optimistic space-based WL, CL, and SN experiments. The table entries are shown using the units of the DETF Report: IM compares favorably with other proposed Stage III projects. The dark energy equation of state is parametrized as $w=w_{p}+\left(a_{p}-a\right) w^{\prime}$. $\Omega_{\mathrm{DE}}$ and $\Omega_{k}$ are the dark energy and curvature density, respectively. $n_{s}$ is the spectral index of the primordial power spectrum, and $A$ its normalization. The Figure of Merit (FoM) refers to the inverse volume in the $95 \%$ confidence ellipse.

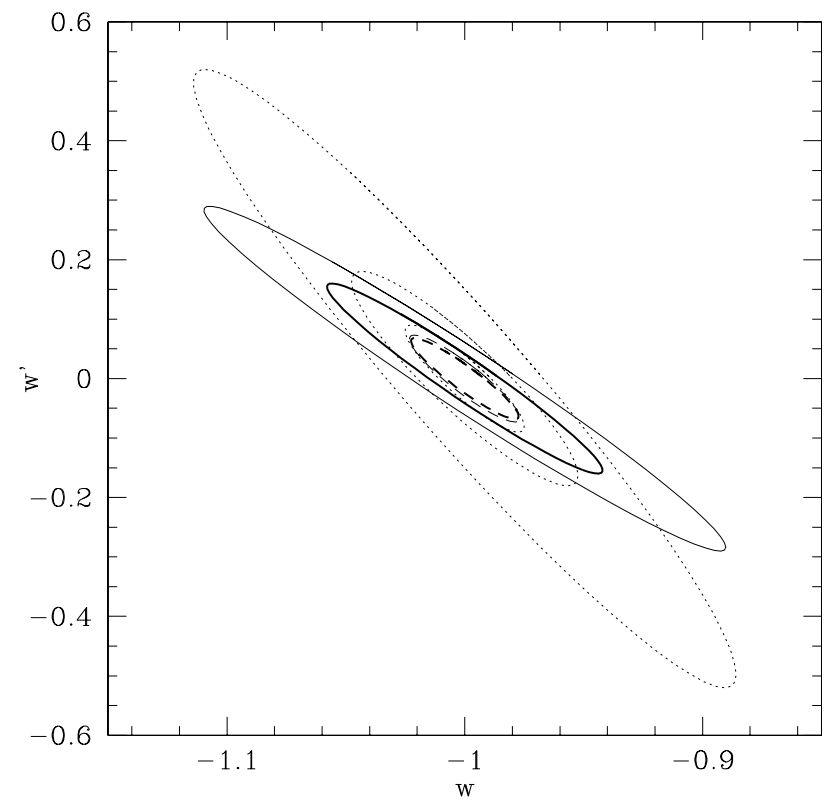

FIG. 4: The 1- $\sigma$ contour for IM combined with Planck (inner thick solid for baseline model, outer thin solid for worst case), the Dark Energy Task Force Stage II projects with Planck (outer dotted), the Stage II and III projects with Planck (intermediate dotted), the Stage II, III and IV projects with Planck (inner dotted), and all above experiments combined (dashed, again thick for baseline, thin for worst case; the two contours are nearly indistinguishable).

three dimensional $21 \mathrm{~cm}$ intensity mapping is an approach that can be tested with existing telescopes today, which appears to offer very competitive constraints on dark energy parameters. This new tool may soon allow astronomers to economically map huge volumes of the universe.

Acknowledgments. - We acknowledge financial support by NSERC.

* Electronic address: tchang@cita.utoronto.ca

$\dagger$ Electronic address: pen@cita.utoronto.ca

‡ Electronic address: jbp@cmu.edu

$\S$ Electronic address: pmcdonal@cita.utoronto.ca

[1] C. Blake and K. Glazebrook, Astrophys. J. 594, 665 (2003); H.-J. Seo and D. Eisenstein, Astrophys. J. 598, 720 (2003); F. B. Abdalla and S. Rawlings, Mon. Not. R. Astron. Soc. 360, 27 (2005); D. Eisenstein, New Astronomy Reviews 49, 360 (2005); A list of proposed BAO experiments includes: ADEPT (http://www.jhu.edu/news_info/news/home06/aug06/ adept.html), DES (http://decam.fnal.gov, http://www.darkenergysurvey.org),

FMOS/WFMOS [astro-ph/0507457], HETDEX (http://www.as.utexas.edu/hetdex), WiggleZ (http://astronomy.swin.edu.au/ ${ }^{\sim}$ karl/KarlHome/Home.html).

[2] S. Cole et al., Mon. Not. R. Astron. Soc. 362, 505 (2005); D. Eisenstein et al., Astrophys. J. 633, 520 (2005); G. Huetsi, Astron. \& Astrophys. 449, 891 (2006); W.J. Percival et al., 2007, astro-ph/0704.3991.

[3] E. Kolb et al., 2006, http://www.nsf.gov/mps/ast/aaac/ dark_energy_task_force/report/detf_final_report.pdf.

[4] D. Spergel et al., Astrophys. J. Suppl. 170, 377 (2007).

[5] S. Wyithe, A. Loeb, and P. Geil, astro-ph/0709.2955.

[6] A. Wolfe, E. Gawiser, and J. X. Prochaska, Annu. Rev. Astron. Astrophys. 43, 861 (2005); S. M. Rao, D. A. Turnshek, and D. B. Nestor, Astrophysical J. 636, 610 
(2006).

[7] R. Barkana and A. Loeb, Rep. Prog. Phys. 70, 627 (2007).

[8] N. Kaiser, Mon. Not. R. Astr. Soc. 222, 323 (1986).

[9] N. Kaiser, Mon. Not. R. Astr. Soc. 227, 1 (1987).

[10] D. Eisenstein and W. Hu, Astrophys. J. 496, 605 (1998).

[11] J.B. Peterson, K. Bandura and U. Pen, astro$\mathrm{ph} / 0606104$.

[12] S. Furlanetto, S.P. Oh and F. Briggs, Phys. Rept. 433,
$181(2006)$

[13] A. Kogut et al., astro-ph/0704.3991.

[14] H.-J. Seo and D. Eisenstein, astro-ph/0701079.

[15] M.A. Zwaan, M.J. Meyer, L. Staveley-Smith, and R.L. Webster, Mon. Not. R. Astr. Soc. 359, 30 (2005).

[16] D. Eisenstein, H.-J. Seo, S. Edwin, D.N. Spergel, Astrophys. J. 664, 675 (2007). 